\title{
Can we avoid dose escalation for intermediate- risk prostate cancer in the setting of short-course neoadjuvant androgen deprivation?
}

This article was published in the following Dove Press journal:

OncoTargets and Therapy

17 March 2016

Number of times this article has been viewed

\author{
Thomas P Shakespeare ${ }^{1,2}$ \\ Shea W Wilcox' \\ Noel J Aherne ${ }^{1,2}$ \\ 'Department of Radiation Oncology, \\ North Coast Cancer Institute, \\ ${ }^{2}$ Faculty of Medicine, Rural Clinical \\ School, The University of New South \\ Wales, Coffs Harbour, New South \\ Wales, Australia
}

Background: Both dose-escalated external beam radiotherapy (DE-EBRT) and androgen deprivation therapy (ADT) improve the outcomes in patients with intermediate-risk prostate cancer. Despite this, there are only few reports evaluating DE-EBRT for patients with intermediaterisk prostate cancer receiving neoadjuvant ADT, and virtually no studies investigating dose escalation $>74$ Gy in this setting. We aimed to determine whether DE-EBRT $>74$ Gy improved the outcomes for patients with intermediate-risk prostate cancer who received neoadjuvant ADT.

Findings: In our institution, patients with intermediate-risk prostate cancer were treated with neoadjuvant ADT and DE-EBRT, with doses sequentially increasing from 74 Gy to 76 Gy and then to 78 Gy between 2006 and 2012. We identified 435 patients treated with DE-EBRT and ADT, with a median follow-up of 70 months. For the $74 \mathrm{~Gy}, 76 \mathrm{~Gy}$, and 78 Gy groups, five-year biochemical disease-free survival rates were $95.0 \%, 97.8 \%$, and $95.3 \%$, respectively; metastasis-free survival rates were $99.1 \%, 100.0 \%$, and $98.6 \%$, respectively; and prostate cancerspecific survival rate was $100 \%$ for all three dose levels. There was no significant benefit for dose escalation either on univariate or multivariate analysis for any outcome.

Conclusion: There was no benefit for DE-EBRT $>74$ Gy in our cohort of intermediate-risk prostate cancer patients treated with neoadjuvant ADT. Given the higher risks of toxicity associated with dose escalation, it may be feasible to omit dose escalation in this group of patients. Randomized studies evaluating dose de-escalation should be considered.

Keywords: radiotherapy, IMRT, dose, dose escalation, dose de-escalation, androgen deprivation therapy, prostate cancer

\section{Introduction}

Meta-analyses demonstrate that both dose-escalated external beam radiotherapy $(\mathrm{DE}-\mathrm{EBRT})^{1}$ and androgen deprivation therapy (ADT) combined with radiotherapy ${ }^{2}$ improve prostate cancer outcomes. However, it has been noted that there is little evidence supporting the use of dose escalation in patients also receiving ADT. ${ }^{3}$

Only two randomized trials have evaluated DE-EBRT in patients receiving ADT. ${ }^{4,5}$ Both studies compared 64 Gy EBRT with 74 Gy DE-EBRT, with all patients receiving neoadjuvant ADT. Neither study reported a significant benefit for dose escalation in the intermediate-risk group. It is thus not surprising that others have questioned the benefit of DE-EBRT for patients receiving ADT. ${ }^{3,6}$

Several randomized trials show that in the absence of ADT, EBRT doses $>70$ Gy provide some benefits, ${ }^{7-10}$ with several national guidelines recommending doses up to 78-81 Gy for intermediate-risk patients. ${ }^{11-13}$ All these guidelines recommend consideration of neoadjuvant ADT with DE-EBRT. However, there is virtually no
Correspondence: Thomas P Shakespeare Department of Radiation Oncology, North Coast Cancer Institute, 345 Pacific Highway, Coffs Harbour, NSW 2450, Australia

Tel +6I 66565125

Fax +6166565128

Email thomas.shakespeare@ncahs.health. nsw.gov.au
OncoTargets and Therapy 2016:9 1635-1639 Dovepress http://dx.doi.org/1 $0.2147 /$ OTT.S1 02327 (c) (1) (-) 2016 Shakespeare et al. This work is published and licensed by Dove Medical Press Limited. The full terms of this license are available at https://www.dovepress.com/terms.php cc) ${ }_{\mathrm{BY}} \mathrm{NC}$ and incorporate the Creative Commons Attribution - Non Commercial (unported, v3.0) License (http:///creativecommons.org/licenses/by-nc/3.0/). By accessing the work you hereby accept the Terms. Non-commercial uses of the work are permitted without any further permission from Dove Medical Press Limited, provided the work is properly attributed. For permission for commercial use of this work, please see paragraphs 4.2 and 5 of our Terms (https://www.dovepress.com/terms.php). 
evidence for dose-escalating radiotherapy to these levels in the setting of ADT.

Given the lack of available evidence, we evaluated whether dose escalation up to 78 Gy had any advantage in terms of prostate-specific antigen (PSA) disease-free survival, metastasis-free survival (MFS), or prostate cancer-specific survival (PCaSS) outcomes in our cohort of intermediate-risk patients treated exclusively with neoadjuvant ADT and dose-escalated intensity-modulated radiotherapy (IMRT).

\section{Methods}

From 2006, the North Coast Cancer Institute implemented a dose-escalation program for patients with localized prostate cancer. Patients were initially treated with 74 Gy, which was escalated to 76 Gy in 2008, then 78 Gy from 2009 until 2012. All patients were treated with either three-dimensional EBRT or IMRT, as has been previously reported. ${ }^{14,15}$ In brief, after institutional ethics approval (North Coast New South Wales Human Research Ethics Committee, Reference Number QA 101), the electronic medical record of our institution (Mosaiq; Elekta, Crawley, UK) was interrogated to identify all patients with National Comprehensive Cancer Network (NCCN)defined intermediate-risk prostate cancer $^{13}$ treated with our standard protocol of DE-EBRT and ADT. Exclusion criteria included patients postprostatectomy, NCCN low or high risk, node positive, metastatic, histology other than adenocarcinoma, did not receive ADT, or treated from 2013 onward.

All patients received ADT using leuprorelin or goserelin acetate monotherapy (using 3- or 4-month depots), with 3-6 months of neoadjuvant/concurrent ADT. Over $90 \%$ of patients received ADT for 6 months. Patients underwent transrectal ultrasound-guided insertion of fiducial markers followed by magnetic resonance imaging/computed tomography (CT) fusion as previously reported, ${ }^{16}$ unless contraindicated. Patients were treated on our "Bowel and Bladder Protocol," involving low-residue diet, aperients, and a pretreatment oral fluid regimen to achieve a comfortably full bladder and empty rectum. The planning CT ( $2 \mathrm{~mm}$ slices) was performed with patients supine and immobilized with ankle stocks. The prostate and proximal seminal vesicles measuring 4-8 $\mathrm{mm}$ were included in the clinical target volume. The clinical target volume to planning target volume expansion was $5 \mathrm{~mm}$. Patients were treated using a 7- to 9-field IMRT technique with the angles optimized to achieve target coverage and organ at risk sparing. The planning target volume was treated with 73.8-78 Gy in 1.8-2.0 Gy fractions prescribed to the reference point. Image guidance used either daily online $\mathrm{kV}$ portal images (matched to fiducial markers) or daily cone beam CT. Patients without fiducial markers $(<1 \%$ of all patients) underwent daily cone beam CT matching to soft tissue and bone. Patients were followed up by the treating radiation oncologists, with data prospectively recorded in Mosaiq. Biochemical failure was determined using the Phoenix definition (PSA nadir $+2 \mathrm{ng} / \mathrm{mL}$ ). All patients with biochemical failure were restaged with $\mathrm{CT}$ and bone scans, and more recently prostate-specific membrane antigen (PSMA) positron emission tomography (PET). ${ }^{17}$ Salvage ADT was initiated when the PSA reached $10-20 \mathrm{ng} / \mathrm{mL}$ or with documented metastatic disease.

Data were analyzed using SPSS Version 19 (IBM Corporation, Armonk, NY, USA). Biochemical disease-free survival (bDFS), MFS, and PCaSS were calculated with Kaplan-Meier curves, and the log-rank (Mantel-Cox) test was used to compare survival between groups. Follow-up time was calculated from the date of commencement of ADT as recommended by Denham et al. ${ }^{18}$ Univariate analysis was done to assess the relationship between potential prognostic factors and bDFS, MFS, and PCaSS. The variables included were age ( $\leq 70 \mathrm{vs}>70)$, pretreatment PSA $(<10 \mathrm{ng} / \mathrm{mL}$ vs $10 \mathrm{ng} / \mathrm{mL}$ or higher), Gleason score $(3+3=6 / 3+4=7 \mathrm{vs} 4+3=7)$, use of IMRT (no vs yes), and radiation dose (73.8-74 Gy, $76 \mathrm{~Gy}$, and $78 \mathrm{~Gy}$ ). All hazard ratios were calculated with Cox proportional hazard models and expressed relative to the control group. $P$-values were two tailed and considered statistically significant if $<0.05$.

\section{Results}

In total, there were 435 intermediate-risk patients treated with DE-EBRT and ADT, with a median follow-up of 70 months. Patient demographics are shown in Table 1.

Table I Patient characteristics

\begin{tabular}{ll}
\hline $\begin{array}{l}\text { Age (in years) } \\
\text { Median }\end{array}$ & 70 \\
Range & $50-85$ \\
Gleason score & \\
$3+3=6$ & $34(7.8 \%)$ \\
$3+4=7$ & $226(52.0 \%)$ \\
$4+3=7$ & $175(40.2 \%)$ \\
IMRT & \\
No & $5(1.1 \%)$ \\
Yes & $430(98.9 \%)$ \\
Dose & \\
74 Gy & $128(29 \%)$ \\
76 Gy & $47(10.8 \%)$ \\
78 Gy & $260(59.8 \%)$ \\
PSA (ng/ml) & \\
Median & 9.0 \\
Range & $0.8-20.0$ \\
\hline
\end{tabular}

Abbreviations: IMRT, intensity-modulated radiotherapy; PSA, prostate-specific antigen. 
The 5-year bDFS for all 435 patients was 95.5\%. The bDFS rates for $74 \mathrm{~Gy}, 76 \mathrm{~Gy}$, and $78 \mathrm{~Gy}$, respectively, were 95.0\%, 97.8\%, and 95.3\% (Kaplan-Meier curves shown in Figure 1). These differences were not statistically significant $(P=0.2)$.

On both univariate and multivariate analyses, only the PSA level was significant for bDFS (PSA $>10 \mathrm{ng} / \mathrm{ml}$ doing worse, $P=0.01$, hazard ratio 2.9 , 95\% CI 1.3-6.5). Dose levels had no impact on bDFS. Further analyses comparing 74-76 Gy (grouped) vs 78 Gy and comparing 74 Gy vs 76-78 Gy (grouped) showed no impact of dose on bDFS, and PSA remained the only significant variable in the Cox regression analysis.

Five-year MFS was 98.9\%. The MFS rates for $74 \mathrm{~Gy}$, $76 \mathrm{~Gy}$, and $78 \mathrm{~Gy}$, respectively, were $99.1 \%, 100.0 \%$, and 98.6\%. Dose had no impact on MFS $(P=0.6)$. Five-year PCaSS was $100.0 \%$ for all dose levels.

\section{Discussion}

Dose escalation improves bDFS for patients with localized prostate cancer, ${ }^{1}$ whereas ADT not only improves bDFS but also PCaSS and overall survival (OS).,19 This has led some to question the need for dose escalation in patients receiving ADT. ${ }^{3,6}$ Few studies have investigated this issue. Only two randomized studies have been reported, ${ }^{4,5}$ which are limited by the fact that doses were only escalated to $74 \mathrm{~Gy}$. Creak et $\mathrm{al}^{5}$ found a nonstatistically significant trend for PSA control favoring dose escalation but did not analyze for intermediate-risk patients. Dearnaley et $\mathrm{al}^{4}$ did find a significant progression-free survival benefit for DE-EBRT

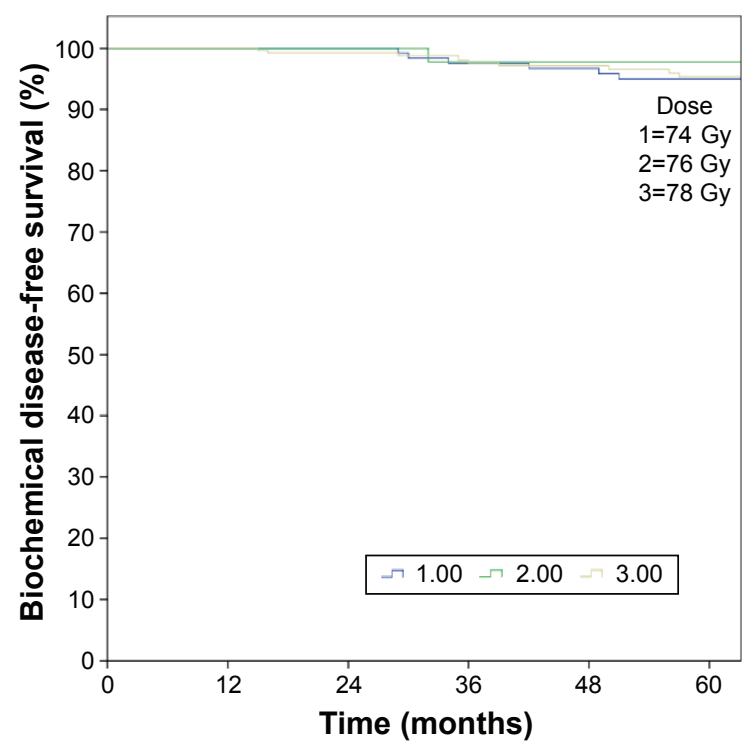

Figure I Biochemical disease-free survival for various dose levels. in the whole cohort of patients; however, the favorable trend was not statistically significant for the intermediate-risk subgroup.

The few other studies evaluating dose escalation in the setting of ADT do not analyze the results for intermediaterisk patients. For example, Denham et $\mathrm{al}^{20}$ conducted a nonrandomized evaluation of EBRT dose levels up to 74 Gy and 46 Gy EBRT combined with high dose rate (HDR) brachytherapy. Higher dose reduced local progression, with no analysis of bDFS. There were 207 intermediate-risk patients (only 19 in the HDR group), however, the results were not analyzed for this risk group.

Stoyanova et $\mathrm{al}^{6}$ reviewed the relative benefits of ADT and DE-EBRT up to $80 \mathrm{~Gy}$, but they did not report the results of the risk group. The results did enable a conclusion that the benefit of ADT far outweighed the benefit of dose escalation. These views have been echoed by Roach, ${ }^{3}$ who also attempted to address the issue of dose escalation vs ADT. With a paucity of high-quality studies, he concluded that the data supporting ADT were greater than the data for dose escalation.

We report one of the largest series of patients with intermediate-risk prostate cancer treated exclusively with a combination of neoadjuvant ADT and DE-EBRT. To our knowledge, this is the only study evaluating the potential benefit of dose escalation $>74$ Gy in the setting of neoadjuvant ADT and EBRT in the management of this risk group. With 70-month median follow-up, we failed to demonstrate any statistically or clinically significant benefit for dose escalation up to 78 Gy. This seems to be consistent with the previous reports comparing $64 \mathrm{~Gy}$ and $74 \mathrm{~Gy} .{ }^{4,5}$

If dose escalation has a questionable benefit in terms of cancer outcomes, it has well-documented adverse effects. The only meta-analysis of randomized trials of dose escalation with long-term follow-up showed that dose escalation increases both late genitourinary and gastrointestinal toxicity. ${ }^{1}$ It is true that ADT has its own toxicities; however, many of these toxicities are reversible, particularly after cessation of short neoadjuvant courses. ${ }^{21}$ Indeed in some studies, ADT has been found to protect against both late radiotherapy-induced urinary and bowel toxicity, ${ }^{21,22}$ perhaps in part due to reduction in prostate size, reducing bladder and rectal dose. ${ }^{23}$

If short-course ADT is more beneficial than dose escalation, it may be possible to limit dose escalation or even dose de-escalate (eg, 60-70 Gy). Similar concepts have been demonstrated in other cancer types, where systemic therapy has allowed radiotherapy doses to be reduced. ${ }^{24,25}$ We believe that further research should be directed to the relative benefits 
of high radiotherapy doses in the setting of neoadjuvant ADT for intermediate-risk prostate cancer.

There are several limitations in our study. First, it is a retrospective review and thus should be regarded as hypothesis generating. We report with a median follow-up of 70 months, and it is possible that significant benefits of dose escalation might occur only with longer follow-up. We also do not report on doses $>78 \mathrm{~Gy}$, and it is possible that benefits may only be seen with much higher doses. We also note that PSMA PET imaging was only available for the most recent follow-up period ${ }^{17}$ and that may have unknown effects on the outcomes. Finally, we have not reported toxicity, with the unknown interactions between escalating doses and ADT, which requires further study.

\section{Conclusion}

In conclusion, for intermediate-risk prostate cancer patients receiving neoadjuvant $\mathrm{ADT}$, we found no benefit for escalating doses up to 78 Gy. There is a lack of evidence for dose escalation in the setting of ADT, and it is apparent that further investigation is required. We hope that our results provide impetus for studies of dose escalation and dose de-escalation, in this group of patients.

\section{Author contributions}

TPS participated in the design of the study, data collection, performed the statistical analysis, and helped draft the manuscript. SWW and NJA participated in data collection and helped draft the manuscript. All authors read and approved the final manuscript.

\section{Disclosure}

The authors report no conflicts of interest in this work.

\section{References}

1. Hou Z, Li G, Bai S. High dose versus conventional dose in external beam radiotherapy of prostate cancer: a meta-analysis of long-term follow-up. J Cancer Res Clin Oncol. 2015;141:1063-1071.

2. Schmidt-Hansen M, Hoskin P, Kirkbride P, Hasler E, Bromham N. Hormone and radiotherapy versus hormone or radiotherapy alone for non-metastatic prostate cancer: a systematic review with meta-analyses. Clin Oncol (R Coll Radiol). 2014;26:e21-e46.

3. Roach M. Dose escalated external beam radiotherapy versus neoadjuvant androgen deprivation therapy and conventional dose external beam radiotherapy for clinically localized prostate cancer: do we need both? Strahlenther Onkol. 2007;183:26-28.

4. Dearnaley DP, Jovic G, Syndikus I, et al. Escalated-dose versus controldose conformal radiotherapy for prostate cancer: long-term results from the MRC RT01 randomised controlled trial. Lancet Oncol. 2014; 15:464-473.

5. Creak A, Hall E, Horwich A, et al. Randomised pilot study of dose escalation using conformal radiotherapy in prostate cancer: long-term follow-up. Br J Cancer. 2013;109:651-657.
6. Stoyanova R, Pahlajani NH, Egleston BL, et al. The impact of doseescalated radiotherapy plus androgen deprivation for prostate cancer using 2 linked nomograms. Cancer. 2013;119:1080-1088.

7. Heemsbergen WD, Al-Mamgani A, Slot A, Dielwart MF, Lebesque JV. Long-term results of the Dutch randomized prostate cancer trial: impact of dose-escalation on local, biochemical, clinical failure, and survival. Radiother Oncol. 2013;110(1):104-109.

8. Kuban DA, Levy LB, Cheung MR, et al. Long-term failure patterns and survival in a randomized dose-escalation trial for prostate cancer. Who dies of disease? Int J Radiat Oncol Biol Phys. 2011;79(5): 1310-1317.

9. Zietman AL, Bae K, Slater JD, et al. Randomized trial comparing conventional-dose with high-dose conformal radiation therapy in earlystage adenocarcinoma of the prostate: long-term results from proton radiation oncology group American college of radiology 95-09. J Clin Oncol. 2010;28(7):1106-1111.

10. Beckendorf V, Guerif S, Le Prise E, et al. 70 Gy versus 80 Gy in localized prostate cancer: 5-year results of GETUG 06 randomized trial. Int J Radiat Oncol Biol Phys. 2011;80(4):1056-1063.

11. Radiotherapy Dose-Fractionation [webpage on the Internet]. The Royal College of Radiologists Board of Faculty of Clinical Oncology. Available from: https://www.rcr.ac.uk/sites/default/files/publication/ Dose-Fractionation_Final.pdf. Accessed November 29, 2015.

12. Cancer Institute NSW [webpage on the Internet]. Intermediate Risk Prostate Cancer Protocol. Available from: https://www.eviq.org.au/ Protocol/tabid/66/categoryid/185/id/234/Radiation+Oncology $\% 2 \mathrm{c}+$ Prostate $\% 2 c+$ Intermediate+Risk $\% 2 c+E B R T \% 2 c+$ Definitive. aspx. Accessed November 29, 2015.

13. NCCN [webpage on the Internet]. Clinical Practice Guidelines in Oncology. Version 1.2016, 11/10/15. National Comprehensive Cancer Network. Available from: http://www.nccn.org/professionals/ physician_gls/pdf/prostate.pdf. Accessed November 29, 2015.

14. Wilcox SW, Aherne NJ, McLachlan CS, McKay MJ, Last AJ, Shakespeare TP. Is modern external beam radiotherapy with androgen deprivation therapy still a viable alternative for prostate cancer in an era of robotic surgery and brachytherapy: a comparison of Australian series. J Med Imaging Radiat Oncol. 2015;59:125-133.

15. Wilcox SW, Aherne NJ, Benjamin LC, et al. Long-term outcomes from dose-escalated image-guided intensity-modulated radiotherapy with androgen deprivation: encouraging results for intermediate- and high-risk prostate cancer. Onco Targets Ther. 2014;7: 1519-1523.

16. Horsley PJ, Aherne NJ, Edwards GV, et al. Planning magnetic resonance imaging for prostate cancer intensity-modulated radiation therapy: impact on target volumes, radiotherapy dose and androgen deprivation administration. Asia Pac J Clin Oncol. 2014;11: $15-21$.

17. Shakespeare TP. Effect of prostate-specific membrane antigen positron emission tomography on the decision-making of radiation oncologists. Radiat Oncol. 2015;10(1):233.

18. Denham JW, Steigler A, Kumar M, et al. Measuring time to biochemical failure in the TROG 96.01 trial: when should the clock start ticking? Int J Radiat Oncol Biol Phys. 2009;75:1008-1012.

19. Jones CU, Hunt D, McGowan DG, et al. Radiotherapy and short-term androgen deprivation for localized prostate cancer. $N$ Engl $J$ Med. 2011;365:107-118.

20. Denham JW, Steigler A, Joseph D, et al. Radiation dose escalation or longer androgen suppression for locally advanced prostate cancer? Data from the TROG 03.04 RADAR trial. Radiother Oncol. 2015; 115:301-307.

21. Denham J, Steigler A, Lamb DS, et al. Short-term androgen deprivation and radiotherapy for locally advanced prostate cancer: results from the Trans-Tasman Radiation Oncology Group 96.01 randomised controlled trial. Lancet Oncol. 2005;6:841-850.

22. Zapatero A, García-Vicente F, Sevillano D, et al. Is hormone therapy a protective factor for late hematuria after high-dose radiotherapy in prostate cancer? Urology. 2008;72:1130-1134. 
23. Samper PM, López Carrizosa MC, Pérez Casas A, et al. Impact of neoadjuvant hormonal therapy on dose-volume histograms in patients with localized prostate cancer under radical radiation therapy. Clin Transl Oncol. 2006;8:599-605.

24. Herskovic A, Martz K, al-Sarraf M, et al. Combined chemotherapy and radiotherapy compared with radiotherapy alone in patients with cancer of the esophagus. N Engl J Med. 1992;326:1593-1598.
25. Sieber M, Rüffer U, Josting A, Diehl V. Treatment of Hodgkin's disease: current strategies of the German Hodgkin's Lymphoma Study Group. Ann Oncol. 1999;10(suppl 6):23-29.

\section{Publish your work in this journal}

OncoTargets and Therapy is an international, peer-reviewed, open access journal focusing on the pathological basis of all cancers, potential targets for therapy and treatment protocols employed to improve the management of cancer patients. The journal also focuses on the impact of management programs and new therapeutic agents and protocols on

\section{Dovepress}

patient perspectives such as quality of life, adherence and satisfaction. The manuscript management system is completely online and includes a very quick and fair peer-review system, which is all easy to use. Visit http://www.dovepress.com/testimonials.php to read real quotes from published authors.

Submit your manuscript here: http://www.dovepress.com/oncotargets-and-therapy-journal 